\title{
Visualizing Excited-State Dynamics of a Diaryl Thiophene: Femtosecond Stimulated Raman Scattering as a Probe of Conjugated Molecules
}

G. Batignani ${ }^{1,2}$, E. Pontecorvo ${ }^{1}$, C. Ferrante ${ }^{1}$, M. Aschi ${ }^{2}$, C. G. Elles ${ }^{3}$, T. Scopigno ${ }^{1,4}$

${ }^{1}$ Dipartimento di Fisica, Universitá di Roma "La Sapienza", I-00185, Roma, Italy

${ }^{2}$ Dipartimento di Scienze Fisiche e Chimiche,

Universitá degli Studi dell'Aquila , I-67100, L'Aquila, Italy

${ }^{3}$ Department of Chemistry, University of Kansas,

Lawrence, Kansas-66045, USA and

${ }^{4}$ Center for Life Nano Science@Sapienza, Istituto Italiano di Tecnologia,

295 Viale Regina Elena, I-00161, Roma, Italy

(Dated: July 13, 2016) 
Franck-Condon dynamics The ultrafast internal vibrational relaxation of the wavepacket towards the $S_{1}$ PES local minimum has been simulated using the Fokker-Planck relation $[1-4]$ :

$$
\frac{\partial \rho}{\partial t}=-\nabla \eta \cdot \boldsymbol{J} \simeq \nabla \eta \cdot\left[\left(\tilde{D}\left(k_{B} T\right)^{-1} \rho \nabla \eta \Delta \mu^{0}+\tilde{D} \nabla \eta \rho\right]\right.
$$

where $\rho$ is the probability distribution of the wavepacket, $\tilde{D}$ indicate the ground state diffusion coefficient and $\Delta \mu^{0}=\Delta \mu^{0}\left(\phi, d_{C-S}\right)$ is the chemical potential along the $S_{1}$ PES. The Fokker-Planck relation has been numerically integrated using a fourth order Runge-Kutta method with 0.1 fs time step. Note that we neither considered the entropic contribution nor any modifications of the molecular volume. Hence, the chemical potential is in this case virtually coincident with the potential energy. In this equation we assume that the diffusion coefficients are time and coordinate independent and also we assume the we do not have off diagonal terms in the matrix of the diffusion coefficients. The initial $\rho_{0}=\rho(t=0)$ distribution has been obtained by a classical molecular dynamics simulation on the ground state (at $300 \mathrm{~K}$, i.e. with the isokinetic temperature coupling), which has been also used to evaluate the diffusion coefficient $\left(\tilde{D}_{d_{C-S}, d_{C-S}}=7 \cdot 10^{-7} \AA^{2} f_{s}^{-1}, \tilde{D}_{\phi, \phi}=0.1 \operatorname{deg}^{2} f^{-1}\right.$ and $\left.\tilde{D}_{d_{C-S}, \phi}=\tilde{D}_{\phi, d_{C-S}}=2 \cdot 10^{-5} \AA \operatorname{deg} f s^{-1}\right)$ via the corresponding computed mean square displacement in time, trough the Einstein relation $\left\langle\left(x(t)-x_{0}\right)\left(y(t)-y_{0}\right)\right\rangle=\left(\tilde{D}_{x y}+\tilde{D}_{y x}\right) t$. Remarkably, the low values of the off-diagonal terms in the diffusion tensor indicate that the cross correlation between the two coordinates does not significantly affect the vibrational relaxation. A video of the wavepacket evolution within the first $4 \mathrm{ps}$ is provided in the Supplementary Material.

Quantifying the timescales of the structural relaxation also allows rationalizing the small $\left(<3 \mathrm{~cm}^{-1}\right)$ experimental shifts in peaks positions observed during this Frank Condon dynamics. In fact, in presence of ultrafast dynamics with relaxation time comparable or shorter than vibrational lifetimes, frequency shifts seen by FSRS are smaller than those of the eigenfrequencies [5]. This is precisely the case here, being $\tau_{1} \approx 1.5 \mathrm{ps}$ ps and $\Gamma^{-1} \approx 1.8 \mathrm{ps}$. Modeling the actinic pump and the Raman probe pulses as impulsive, $E_{A}(t)=E_{A} \delta(t)$, $E_{P}(t)=E_{P} \delta(t-T)$, and assuming a monochromatic Raman pulse $\left(E_{R}(t)=E_{R} e^{-i \omega_{R} t}\right)$ the FSRS spectral responses can be evaluated as

$$
S(\omega)=\Im\left[P^{(5)}(\omega) E_{P}^{*}(\omega)\right]
$$




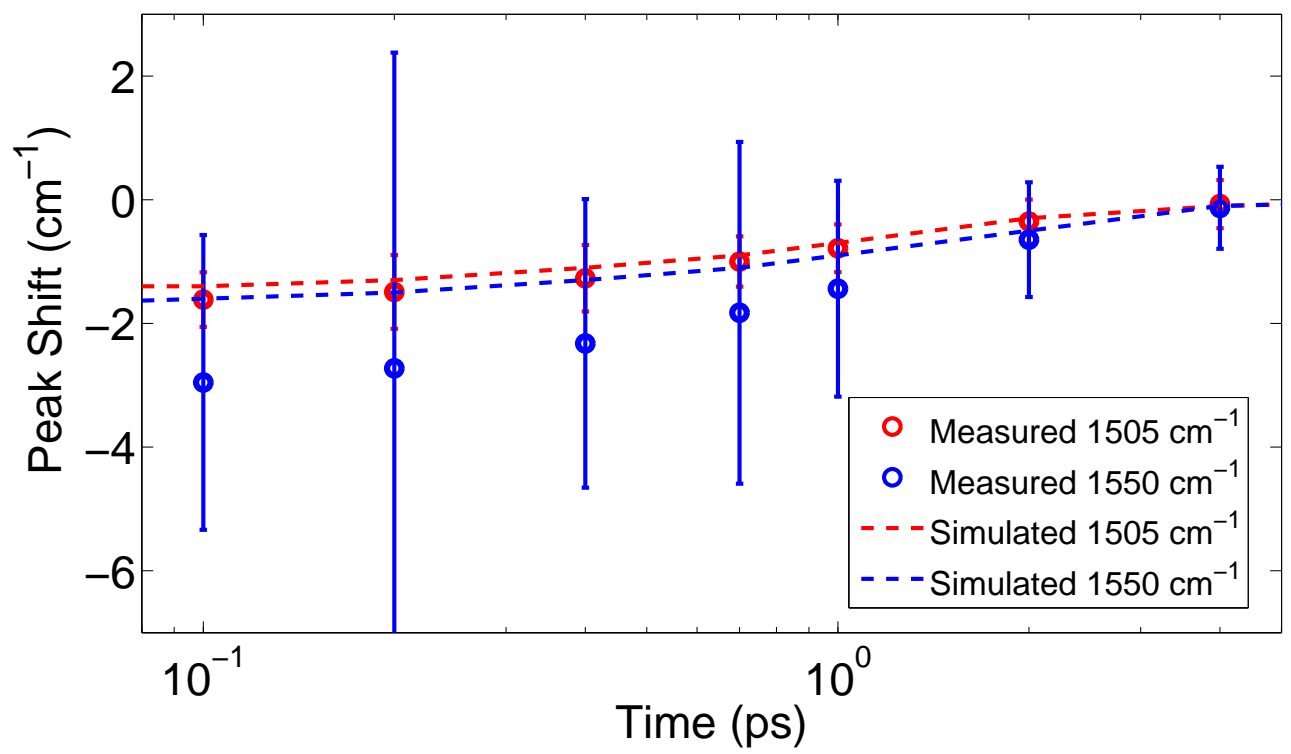

FIG. S1: FSRS measured peak shifts for the $\mathrm{C}=\mathrm{C}$ Raman modes (circles), compared with the simulation based on the $\tau_{1}$ determination (dashed lines) using the eigenfrequencies calculated for the initial and asymptotic geometries along an exponential wavepacket propagation.

where

$$
P^{(5)}(\omega)=\int_{-\infty}^{+\infty} d t P^{(5)}(t) e^{i \omega t}
$$

The nonlinear polarization for a three level system (with initial state at $\omega_{a}$, vibrational excited state at $\omega_{c}$ and electronic resonant excited state at $\omega_{b}$ ) in time-domain then reads:

$$
P^{(5)}(t) \propto i\left|E_{R}\right|^{2} E_{P} \frac{1}{\left(\omega_{R}-\omega_{b a}\right)^{2}} \exp \left[-i \omega_{R}(t-T)+i \int_{T}^{t} \omega_{c a}(\tau) d \tau-\gamma_{c a}|t-T|\right]
$$

where $\gamma_{c a}^{-1}$ and $\omega_{c a}(t)$ indicate the vibrational lifetime and the instantaneous frequency, respectively. The calculated peak shifts, obtained from the maxima of Eq. S2, are reported in Fig. $\mathrm{S} 1$ for the $\mathrm{C}=\mathrm{C}$ stretch vibrations, and turn out to be in good agreement with the FSRS measurements.

\section{Intersystem Crossing on the excited states}

The probability $P^{(L Z)}$ of Diabatic Crossing (DC), along the crossing curve between $S_{1}$ and $T_{2}$ (shown in Fig. S2), can be obtained using the Landau-Zener relation [6]:

$$
P^{(L Z)}=e^{-2 \cdot \pi\left|H_{S O C}\right|^{2} /(h v F)}
$$

where the "reaction coordinate" is the distance between the Carbon and Sulfur atoms and its velocity $v=\frac{d q}{d t}$ has been estimated $\approx 4 \mathrm{~ms}^{-1}$. $H_{S O C}$ are two-electron spin-orbit matrix 
elements and have been computed by Dalton two-electron spin-orbit matrix elements [7], while $\mathrm{F}$ indicates the force $F(d)=F_{S}(d)+F_{T}(d)$, with

$$
F_{S} / D(d)=\left.\frac{d E_{S / T}}{d q}\right|_{d} \approx \frac{E_{S / T}(d+\Delta q)-E_{S / T}(d-\Delta q)}{2 \cdot \Delta q}
$$

where $E_{S / T}(d)$ is the energy of Singlet/Triplet state. In Table S1 we report the computed values for the $H_{S O C}$ matrix elements and for the crossing probability.

TABLE S1: Spin Orbit Coupling Matrix Elements, Derivative of the differential energy surface at different C-S distance and dihedral angle configurations. The corresponding $\mathrm{P}^{(\mathrm{LZ})}$ probability are reported in the last column.

\begin{tabular}{|c|c|c|c|c|}
\hline Distance $(\AA)$ & Dihedral Angle $(\mathrm{deg})$ & $H_{S O C}$ & $F(\mathrm{SI})$ & $\mathrm{P}^{(\mathrm{LZ})}$ \\
\hline 2.05 & 0 & $1.3 \cdot 10^{-4}(\mathrm{Ha})$ & $6.7 \cdot 10^{-9}$ & 0.46 \\
\hline 2.10 & 10 & $1.8 \cdot 10^{-4}(\mathrm{Ha})$ & $4.9 \cdot 10^{-9}$ & 0.14 \\
\hline 2.10 & 20 & $1.8 \cdot 10^{-4}(\mathrm{Ha})$ & $4.1 \cdot 10^{-9}$ & 0.09 \\
\hline 2.10 & 30 & $1.2 \cdot 10^{-4}(\mathrm{Ha})$ & $\approx 1 \cdot 10^{-9}$ & 0.01 \\
\hline 2.15 & 30 & $1.5 \cdot 10^{-4}(\mathrm{Ha})$ & $3.3 \cdot 10^{-10}$ & 0 \\
\hline 2.20 & 20 & $1.8 \cdot 10^{-4}(\mathrm{Ha})$ & $1.57 \cdot 10^{-9}$ & 0.003 \\
\hline 2.20 & 30 & $1.3 \cdot 10^{-4}(\mathrm{Ha})$ & $1.9 \cdot 10^{-9}$ & 0.06 \\
\hline 2.30 & 0 & $9.2 \cdot 10^{-6}(\mathrm{Ha})$ & $8.8 \cdot 10^{-9}$ & 0.99 \\
\hline 2.30 & 10 & $5.7 \cdot 10^{-5}(\mathrm{Ha})$ & $6 \cdot 10^{-9}$ & 0.85 \\
\hline
\end{tabular}

The computed $S_{1}$ MPT normal modes along the reaction coordinates $d_{C-S}$ for $\phi=0^{\circ}$ and $30^{\circ}$ are shown in Fig. S3. The frequencies range for each normal mode, corresponding to the entire $d_{C-S}$ and $\phi$ explored configurational subspace, is shown in Fig. 2.

Peak Position and Intensities In order to obtain peak frequencies and maxima of each mode, FSRS experimental spectra have been fitted using the sum of pseudo-Voigt profiles

$$
S(\omega)=\sum_{n}\left(1-\eta_{n}\right) G_{n}(\omega)+\eta_{n} L_{n}(\omega)
$$

with

$$
G_{n}(\omega)=\frac{\sqrt{4 \ln 2}}{\sqrt{\pi} \sigma_{n}} e^{-\frac{4 \ln 2}{\sigma_{n}^{2}}\left(\omega-\omega_{0}^{n}\right)^{2}}
$$




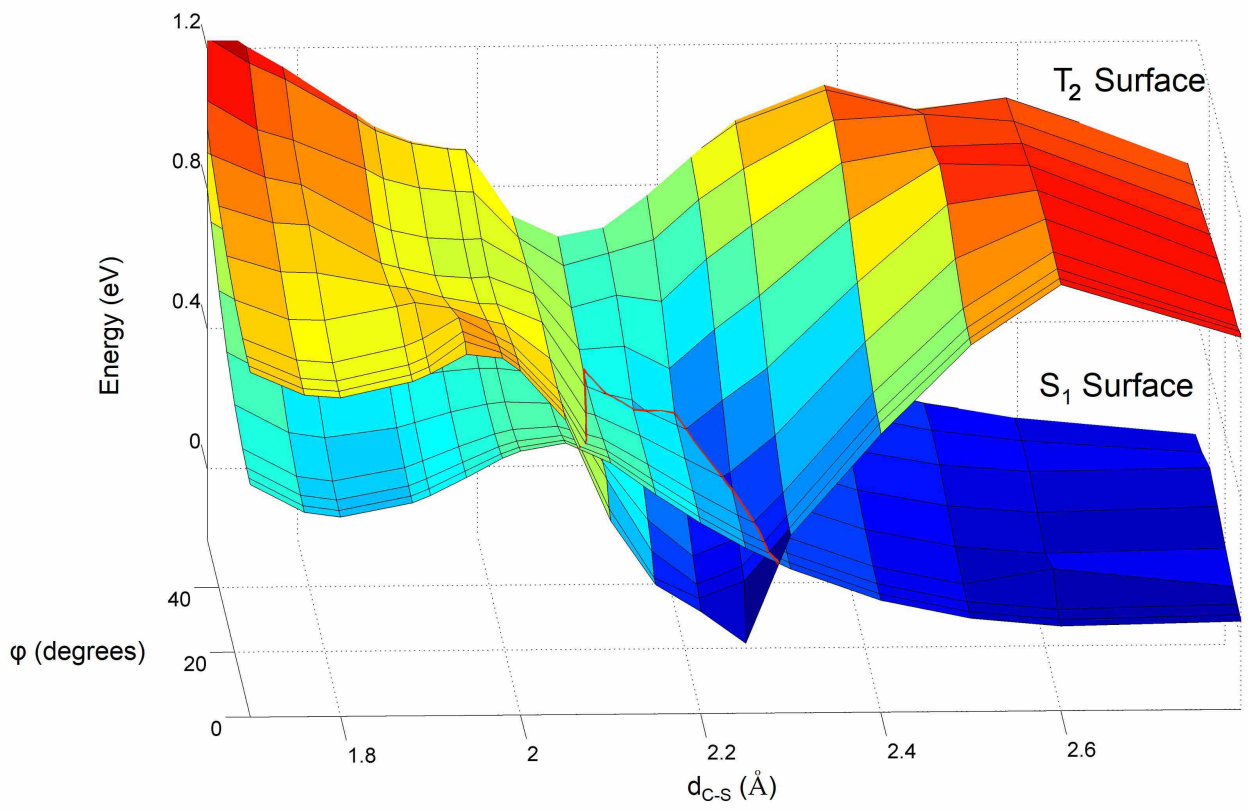

FIG. S2: Intersystem crossing on the Excited Potential Energy Surfaces of Methyl-

Phenylthiophene computed by Time dependent DFT with B3LYP functional and the 6-31+g** basis set. The red line emphasizes the curve of crossing.

and

$$
L_{n}(\omega)=\frac{2}{\pi} \frac{\sigma_{n}}{4\left(\omega-\omega_{0}^{n}\right)+\sigma_{n}^{2}}
$$

where $\omega_{0}^{n}$ and $\sigma_{n}$ indicate respectively the peak frequency and the full width at half maximum of the $n$ Raman mode. With this procedure, we can conveniently isolate overlapping contributions in the high frequency range.

Normal Modes In figure S4, S5, S6 we report sketches of selected MPT normal mode eigenvectors for $S_{0}, S_{1}$ and $T_{1}$, respectively. The normal modes geometrical assignments have been approximately carried out by considering the character of the mode, localized on Phenyl and/or Thiophene ring, adopting for the sake of clarity their own symmetry (i.e. $D_{6 h}$ and $C_{2 v}$, respectively). Hence these assignments should be considered as only indicative.

[1] D. McQuarrie, Statistical mechanics (University Science Books, Sausalito, CA, 2000).

[2] J. D. Bryngelson, J. N. Onuchic, N. D. Socci, and P. G. Wolynes, Proteins: Struct., Funct., Genet. 21, 167 (1995). 

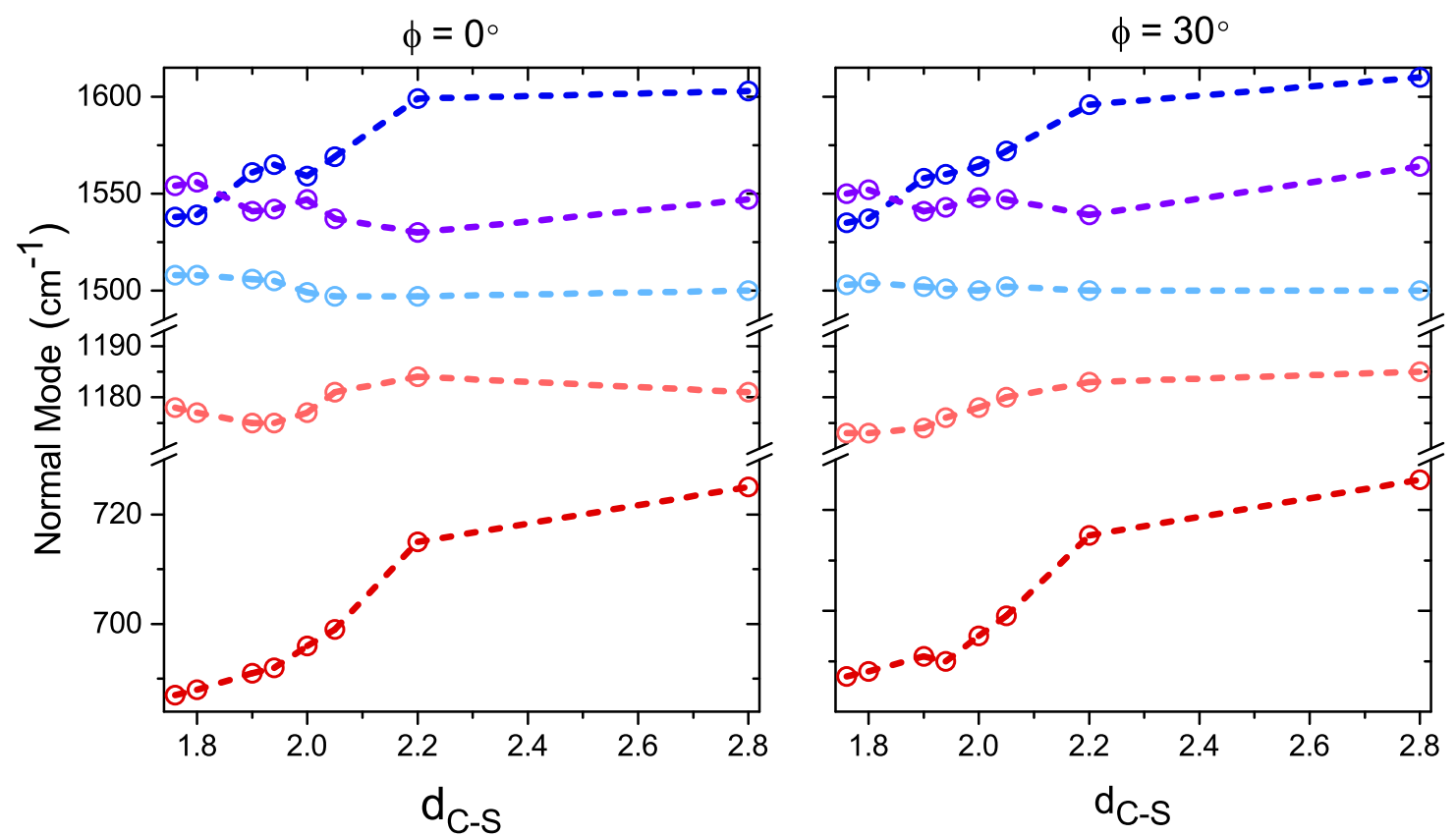

FIG. S3: $S_{1}$ MPT normal modes, observed with FSRS (see Fig. 2), computed along the reaction coordinates $d_{C-S}$ for $\phi=0^{\circ}$ and $\phi=30^{\circ}$.

[3] I. Daidone, M. D'Abramo, A. D. Nola, and A. Amadei, J. Am. Chem. Soc. 127, 14825 (2005).

[4] M. Aschi, M. D’Abramo, F. Ramondo, I. Daidone, M. D’Alessandro, A. D. Nola, and A. Amadei, J. Phys. Org. Chem. 19, 518 (2006).

[5] S. Mukamel and J. D. Biggs, J. Chem. Phys. 134, 161101 (2011).

[6] C. Zener, Proc. R. Soc. A 137, 696 (1932).

[7] K. Aidas, C. Angeli, K. L. Bak, V. Bakken, R. Bast, L. Boman, O. Christiansen, R. Cimiraglia, S. Coriani, P. Dahle, et al., WIREs Comput Mol Sci. 4, 269 (2013). 


\section{$\mathrm{S}_{0}$ Normal Modes}
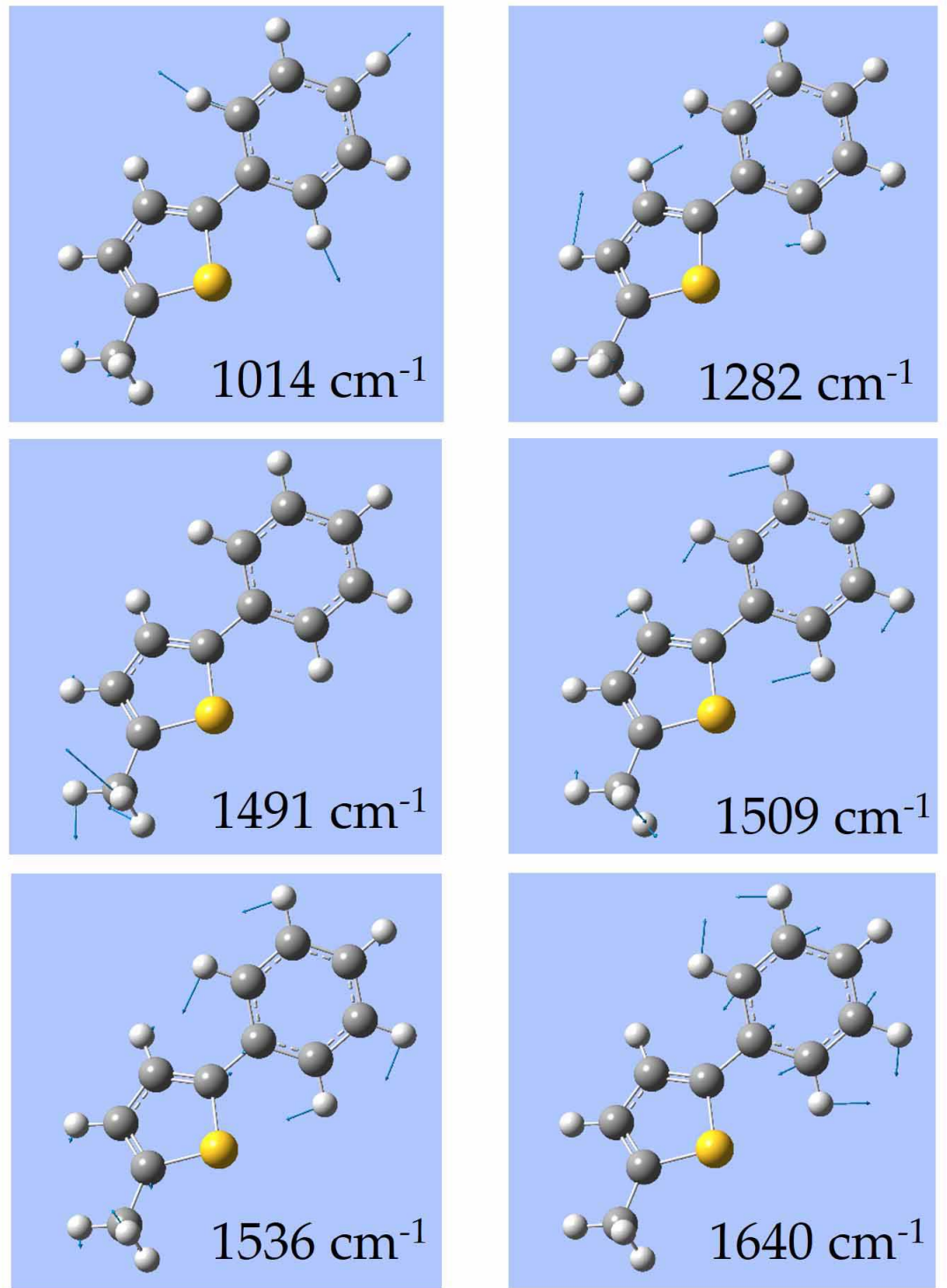

FIG. S4: Selected $S_{0}$ Normal modes computed by DFT with B3LYP functional and the 6$311++\mathrm{g}^{* *}$ basis set. 


\section{$\mathrm{S}_{1}$ Normal Modes}
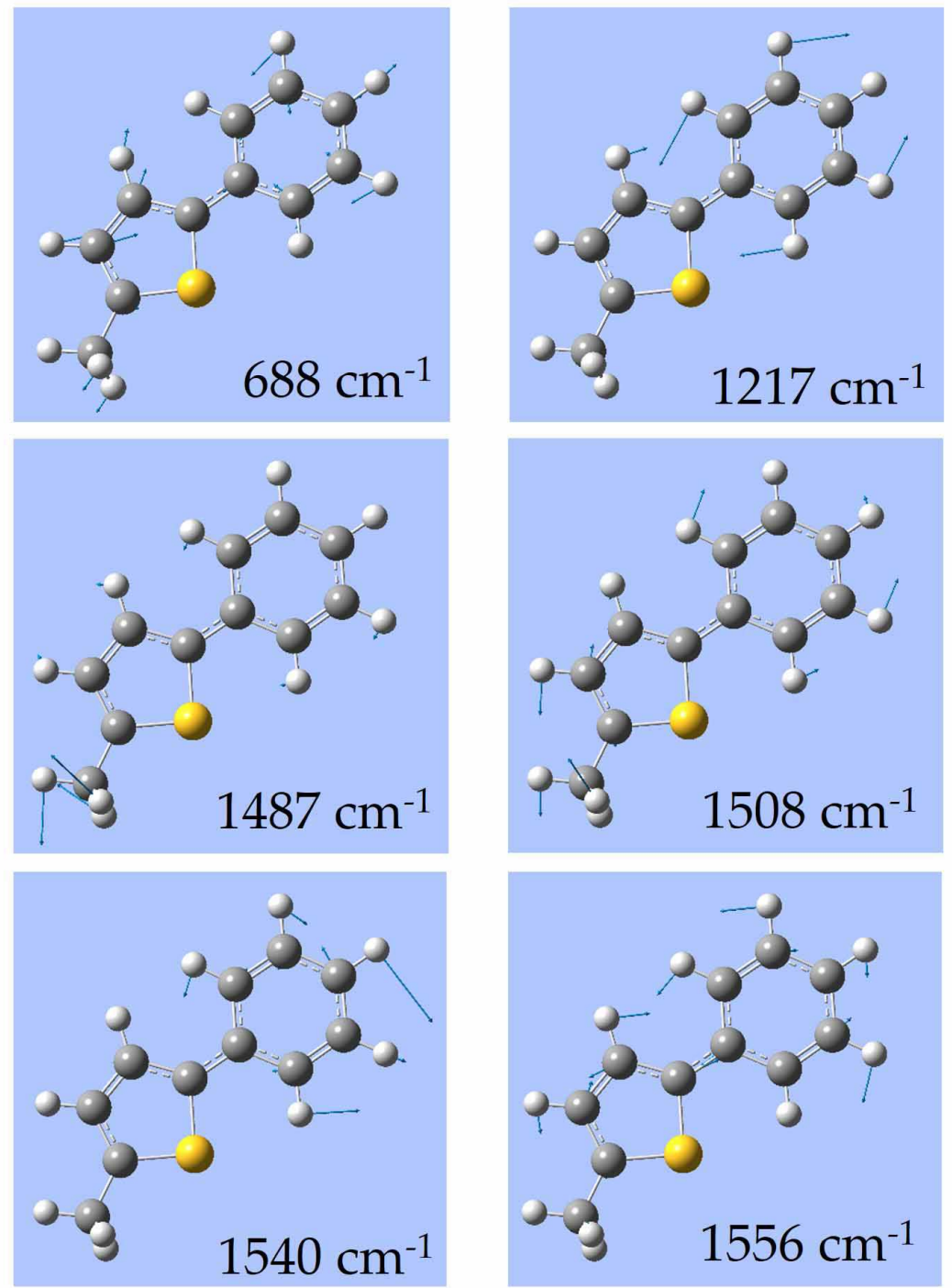

FIG. S5: Selected $S_{1}$ Normal modes computed by DFT with B3LYP functional and the 6$31+\mathrm{g}^{* *}$ basis set. 


\section{$\mathrm{T}_{1}$ Normal Modes}
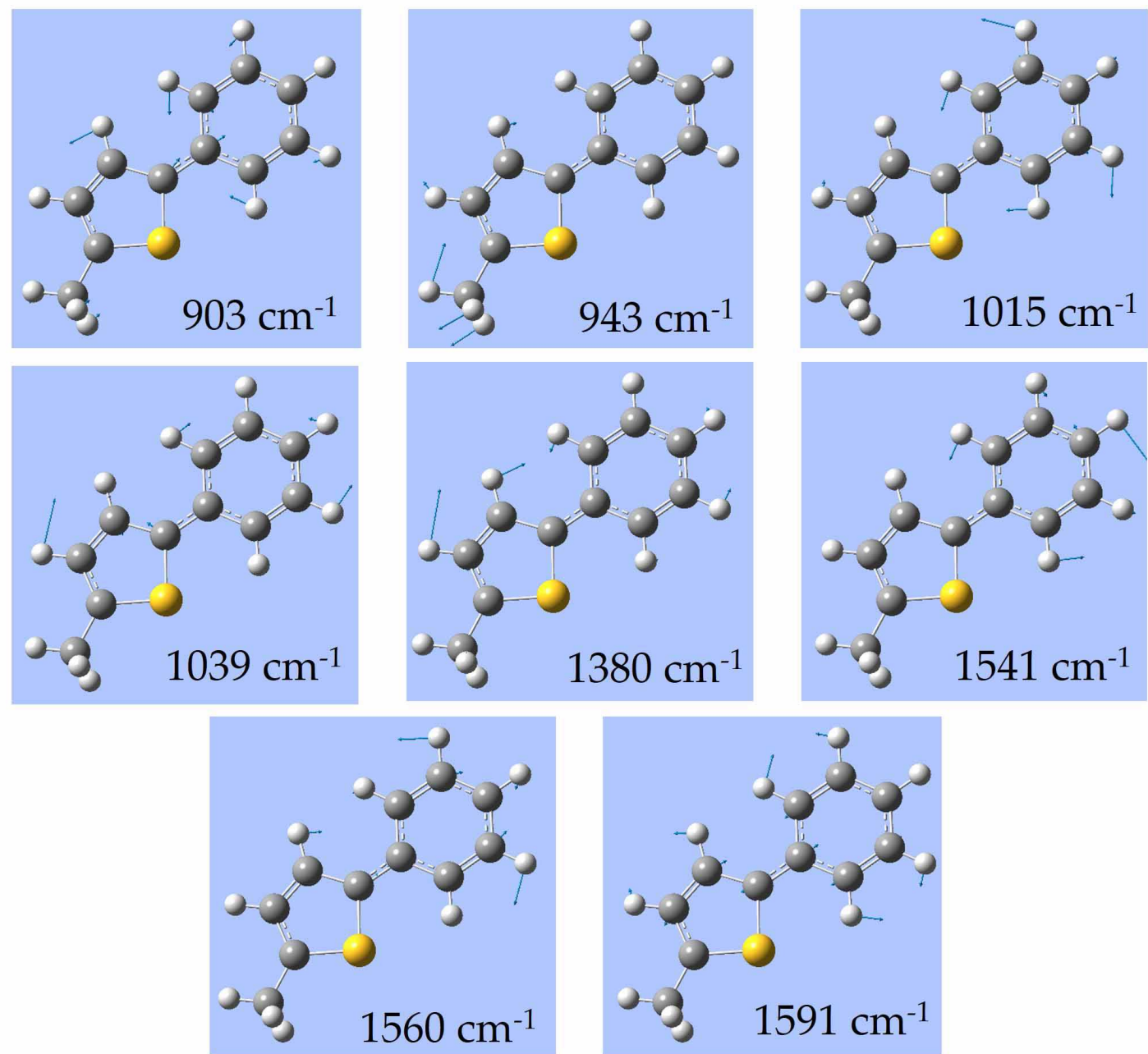

FIG. S6: Selected $T_{1}$ Normal modes computed by DFT with B3LYP functional and the 6$311++\mathrm{g}^{* *}$ basis set. 